\title{
5D World-Universe Model. Gravitation
}

\author{
Vladimir S. Netchitailo \\ Biolase Inc., Irvine, CA, USA \\ Email: v.netchitailo@sbcglobal.net
}

Received 29 March 2016; accepted 1 July 2016; published 5 July 2016

Copyright (C) 2016 by author and Scientific Research Publishing Inc.

This work is licensed under the Creative Commons Attribution International License (CC BY).

http://creativecommons.org/licenses/by/4.0/

(c) (i) Open Access

\section{Abstract}

5D World-Universe Model is based on the decisive role of the Medium of the World composed of massive particles: protons, electrons, photons, neutrinos, and dark matter particles. In this manuscript we discuss different aspects of the gravitation: measured values of the Newtonian parameter of Gravitation and different Gravitational effects (gravitational lensing, cosmological redshift, gravitational deflection of light and gravitational refraction, proposed in the present paper). We show inter-connectivity of all cosmological parameters and provide a mathematical framework that allows direct calculation of them based on the value of the gravitational parameter. We analyze the difference between Electromagnetism and Gravitoelectromagnetism and make a conclusion about the mandatory existence of the Medium of the World. This paper aligns the World-Universe Model with the Le Sage's theory of gravitation and makes a deduction on Gravity, Space and Time be emergent phenomena.

\section{Keywords}

5D World-Universe Model, Newtonian Parameter of Gravitation, Le Sage's Gravity, Cosmic Neutrino Background, Gravitoelectromagnetism, Medium of the World, Cosmological Parameters, Emergent Phenomena

\section{Introduction}

We can't solve problems by using the same kind of thinking we used when we created them.

Albert Einstein

Today, a growing feeling of stagnation is shared by a large number of researchers. In his "The Twilight of the Scientific Age” (2013), Martin Lopez Corredoira outlines the most significant issues he believes, Physics todays’ experiences, as a discipline: increasingly expensive experiments that yield less and less, lack of outstanding results, lack of openness to new ideas exhibited by scientific journals and community as a whole.

In some respects, the situation today is similar to that at the end of $19^{\text {th }}$ century, when the common consensus held that the body of Physics was nearly complete. Discoveries of special and general relativity, quantum me- 
chanics and elementary particles shook that belief and led to a new renaissance in Physics that lasted for a century. The genius of Einstein, Planck, Bohr, Dirac, Heisenberg, and Schrödinger allowed them to propose fundamentally new theories with very little experimental data to back them up.

During the $20^{\text {th }}$ century, their theories were validated and elaborated with newly acquired experimental results. The pendulum may, however, have swung too far: today, all results must be made fit into the existing framework. The frameworks get adjusted when necessary, particularly inconvenient results may even get discarded at times. The time may be ripe to propose new fundamental models that will be both simpler than the current state of the art, as well as open up new areas of research.

In 1937, Paul Dirac proposed a new basis for cosmology: the hypothesis of a variable gravitational "constant"; and later added the notion of continuous creation of matter in the World. In 1983, Paul Wesson developed 5D Space-Time-Mass theory that associated the fifth dimension with rest mass of particles. The gravitational constant serves as the dimension-transposing parameter.

5D World-Universe Model (WUM) follows these ideas, albeit introducing a different mechanism of matter creation. WUM rests on the theoretical basis developed by Prof. Wesson, with the following modifications [1]: the fifth dimension is associated with the total energy of the Medium of the World, and the gravitomagnetic parameter of the Medium serves as the dimension-transposing parameter.

A number of ideas presented in this paper are not new, and I don't claim credit for them. In fact, several ideas belonging to classical physicists such as P. A. M. Dirac, P. S. Wesson, A. D. Sakharov, O. Heaviside, Le Sage, and J. McCullagh, are revisited in a new light.

The 5D WUM is proposed as an alternative to the prevailing Bing Bang Model of standard physical cosmology. The main differences are the existence of the Medium of the World and the source of the World's energy.

WUM analyzes the role of the Intergalactic plasma consisting of protons, electrons, and photons as part of the Medium of the World [1], discusses Multicomponent Dark Matter and its decisive role in the Medium and Macroobjects of the World [2], and considers mass-varying neutrinos as part of the Medium of the World [3].

This paper discusses the Gravitation of the World. In Section 2 we make analysis of the measured values of the Newtonian parameter of Gravitation. In Section 3 we show inter-connectivity of all cosmological parameters and provide a mathematical framework that allows their direct calculation based on the value of the Gravitational parameter. In Section 4 we present different gravitational effects: gravitational lensing, cosmological redshift, gravitational deflection of light and gravitational refraction, proposed in the present paper. The Gravitoelectromagnetism is discussed in Section 5. Le Sage's gravity mechanism is analyzed in Section 6. In Section 7 we deduce on Gravity, Space and Time to be emergent phenomena.

\section{Observations of Newtonian Parameter of Gravitation}

The accuracy of the measured value of Gravitational parameter $G$ has increased only modestly since the original Cavendish experiment. Published values of $G$ have varied rather broadly, and some recent measurements of high precision are, in fact, mutually exclusive.

Table 1, borrowed from CODATA Recommended Values of the Fundamental Physical Constants, 2010, summarizes the results of measurements of the Newtonian parameter of gravitation relevant to the 2010 adjustment [4]:

Observe that the values of $G$ vary significantly depending on method. The disagreement in the values of $G$ obtained by the various teams far exceeds the standard uncertainties provided with the values.

Detailed analysis of the results of measurements of the Newtonian parameter of gravitation in Table 1 shows that there are three groups of measurements. Inside each such group, the measurements are not mutually exclusive; however measurements outside of a group contradict the entire group.

- The first such group consists of six measurements with the average value of

$$
G_{1}=6.67401(19) \times 10^{-11} \mathrm{~m}^{3} \cdot \mathrm{kg}^{-1} \cdot \mathrm{s}^{-2}
$$

and relative standard uncertainty $28.5 \mathrm{ppm}$;

- The second one consists of four measurements with the average value of

$$
G_{2}=6.67250(16) \times 10^{-11} \mathrm{~m}^{3} \cdot \mathrm{kg}^{-1} \cdot \mathrm{s}^{-2}
$$

and relative standard uncertainty $24 \mathrm{ppm}$;

The third one consists of one measurement with the value of 
Table 1. Measurements of Newtonian parameter of gravitation.

\begin{tabular}{|c|c|c|c|c|}
\hline Source & Identification $^{\mathrm{a}}$ & Method & $10^{11} \mathrm{G}$ & Rel. stand. \\
\hline & & & $\mathrm{m}^{3} \cdot \mathrm{kg}^{-1} \cdot \mathrm{s}^{-2}$ & uncert $\mathrm{u}_{\mathbf{r}}$ \\
\hline Luther and Towler (1982) & NIST-82 & $\begin{array}{l}\text { Fiber torsion balance, } \\
\text { dynamic mode }\end{array}$ & $6.67248(43)$ & $6.4 \times 10^{-5}$ \\
\hline Karagioz and Izmailov (1996) & TR\&D-96 & $\begin{array}{l}\text { Fiber torsion balance, } \\
\text { dynamic mode }\end{array}$ & $6.6729(5)$ & $7.5 \times 10^{-5}$ \\
\hline Bagley and Luther (1997) & LANL-97 & $\begin{array}{l}\text { Fiber torsion balance, } \\
\text { dynamic mode }\end{array}$ & $6.67398(70)$ & $1.0 \times 10^{-4}$ \\
\hline Gundlach and Merkowitz $(2000,2002)$ & UWash-00 & $\begin{array}{l}\text { Fiber torsion balance, } \\
\text { dynamic compensation }\end{array}$ & $6.674255(92)$ & $1.4 \times 10^{-5}$ \\
\hline Quinn et al. (2001) & BIPM-01 & $\begin{array}{c}\text { Strip torsion balance, } \\
\text { compensation mode, static deflection }\end{array}$ & 6.675 59(27) & $4.0 \times 10^{-5}$ \\
\hline Kleinevoß (2002); Kleinvoß et al. (2002) & UWup-02 & $\begin{array}{c}\text { Suspended body, } \\
\text { displacement }\end{array}$ & $6.67422(98)$ & $1.5 \times 10^{-4}$ \\
\hline Armstrong and Fitzgerald (2003) & MSL-03 & $\begin{array}{l}\text { Strip torsion balance, } \\
\text { compensation mode }\end{array}$ & $6.67387(27)$ & $4.0 \times 10^{-5}$ \\
\hline Hu et al. (2005) & HUST-05 & $\begin{array}{l}\text { Fiber torsion balance, } \\
\text { dynamic mode }\end{array}$ & $6.67228(87)$ & $1.3 \times 10^{-4}$ \\
\hline Schlamminger et al. (2006) & UZur-06 & $\begin{array}{l}\text { Stationary body, } \\
\text { weight change }\end{array}$ & $6.67425(12)$ & $1.9 \times 10^{-5}$ \\
\hline Luo et al. (2009); Tu et al. (2010) & HUST-09 & $\begin{array}{l}\text { Fiber torsion balance, } \\
\text { dynamic mode }\end{array}$ & $6.67349(18)$ & $2.7 \times 10^{-5}$ \\
\hline Parks and Faller (2010) & JILA-10 & $\begin{array}{c}\text { Suspended body, } \\
\text { displacement }\end{array}$ & $6.67234(14)$ & $2.1 \times 10^{-5}$ \\
\hline
\end{tabular}

${ }^{a}$ NIST: National Institute of Standards and Technology, Gaithersburg, MD, USA; TR \& D: Tribotech Research and Development Company, Moscow, Russian Federation; LANL: Los Alamos National Laboratory, Los Alamos, New Mexico, USA; UWash: University of Washington, Seattle, Washington, USA; BIPM: International Bureau of Weights and Measures, S`evres, France; UWup: University of Wuppertal, Wuppertal, Germany; MSL: Measurement Standards Laboratory, Lower Hutt, New Zeland; HUST: Huazhong University of Science and Technology, Wuhan, PRC; UZur: University of Zurich, Zurich, Switzerland; JILA: JILA, University of Colorado and National Institute of Standards and Technology, Boulder, Colorado, USA.

$$
G_{3}=6.67559(27) \times 10^{-11} \mathrm{~m}^{3} \cdot \mathrm{kg}^{-1} \cdot \mathrm{s}^{-2}
$$

and relative standard uncertainty $40 \mathrm{ppm}$.

Clearly, the relative uncertainty of any such group is better than the uncertainty of the entire result set. $G_{1}, G_{2}$ and $G_{3}$ have relative standard uncertainties that are smaller than the average value of $G$. Out of the three distinct groups of $G$ measurements, how shall we identify the correct one?

In accordance with WUM, the Gravitational parameter $G$ and Fermi coupling parameter $G_{F}$ can be expressed as follows [3]:

$$
\begin{gathered}
G=\frac{a^{2} c^{4}}{8 \pi h c} \times Q^{-1} \\
\frac{G_{F}}{(\hbar c)^{3}}=\sqrt{30}\left(2 \alpha \frac{m_{e}}{m_{p}}\right)^{1 / 4} \times \frac{m_{p}}{m_{e}} \frac{1}{E_{0}^{2}} \times Q^{-1 / 4}
\end{gathered}
$$


where $\hbar$ is Dirac constant, $c$ is the electrodynamic constant, $\alpha$ is fine-structure constant, $m_{p}$ is the mass of a proton, $m_{e}$ is the mass of an electron, and basic energy unit $E_{0}$ equals to

$$
E_{0}=\frac{h c}{a}
$$

where $h=2 \pi \hbar$ is Planck constant, $a_{0}$ is the classical radius of an electron, and $a=2 \pi a_{0}$.

For the three groups of $G$ measurements, parameter $Q$ will take on the following values, respectively (see 2.4):

$$
\begin{gathered}
Q_{1}=0.759981(22) \times 10^{40} \\
Q_{2}=0.760153(18) \times 10^{40} \\
Q_{3}=0.759801(30) \times 10^{40}
\end{gathered}
$$

The calculated value of the parameter $Q_{F}$ (see 2.5) based on the average value of the Fermi coupling parameter $G_{F}=1.1663787(6) \times 10^{-5} \mathrm{GeV}^{-2}$ is:

$$
Q_{F}=0.75992106 \times 10^{40}
$$

The value of $Q_{F}$ is much more precise than the values of $Q_{1}, Q_{2}, Q_{3}$. With this value of $Q_{F}$ we can make the choice of the first group of $G$ measurements and significantly increase the precision of all $Q$-dependent parameters (see Section 3).

The calculated value of the parameter $Q_{G}$ based on the average value of the gravitational parameter $G=6.67408(31) \times 10^{-11} \mathrm{~m}^{3} \cdot \mathrm{kg}^{-1} \cdot \mathrm{s}^{-2} \quad($ CODATA, 2014)

$$
Q_{G}=0.759972 \times 10^{40}
$$

is very close to the value of $Q_{1}$ and correspond to the value of $Q_{F}$. The calculated value of $G$ based on the average value of $G_{F}$

$$
G=6.6745358 \times 10^{-11} \mathrm{~m}^{3} \cdot \mathrm{kg}^{-1} \cdot \mathrm{s}^{-2}
$$

The CODATA, 2014 value of $G$ is slightly smaller $(<0.007 \%)$ than this calculated value.

The gravitational parameter $G$ in our Model is changing in time $G \propto \tau^{-1}$ with the following rate:

$$
\dot{G} / G=7.03 \times 10^{-11} \mathrm{yr}^{-1}
$$

During the 216 years elapsed from the first measurement of the value of $G$ by Henry Cavendish, value of $G$ has decreased by $\Delta G$ :

$$
\Delta G=1.52 \times 10^{-8} \mathrm{~m}^{3} \cdot \mathrm{kg}^{-1} \cdot \mathrm{s}^{-2}
$$

The above $\Delta G$ is far smaller than the precision that we have attained when measuring $G$, and thus measuring $\Delta G$ directly seems to be impossible using contemporary techniques.

In his papers Jean-Philippe Uzan reviewed the main experimental and observational constraints that have been obtained for variations of the gravitational parameter in different areas [5] [6]:

- Solar systems constraints,

- Pulsar timing,

- Stellar constraints,

- Cosmological constraints, and found that

$$
\dot{G} / G \lesssim 10^{-11} \Leftrightarrow 10^{-12} \mathrm{yr}^{-1}
$$

The experimentally obtained constraints on $G$ variation rates are significantly larger than theoretically calculated 2.13. Note that all obtained constraints are the results of the calculations based on different theoretical models. One example from review [6]:

"The Lunar Laser Ranging (LLR) experiment has measured the relative position of the Moon with respect to the Earth with accuracy of the order of $1 \mathrm{~cm}$ over 3 decades. An early analysis of this data assuming a Brans-Dicke theory of gravitation gave that $\dot{G} / G \leq 3 \times 10^{-11} \mathrm{yr}^{-1}$. It was improved by using 20 years of observation to get 
$\dot{G} / G \leq 1.04 \times 10^{-11} \mathrm{yr}^{-1}$, the main uncertainty arising from Lunar tidal acceleration. With 24 years of data, one reached $\dot{G} / G \leq 6 \times 10^{-12} \mathrm{yr}^{-1}$ and finally, the latest analysis of the Lunar laser ranging experiment increased the constraint to

$$
\dot{G} / G \leq(4 \pm 9) \times 10^{-13} \mathrm{yr}^{-1}
$$

Another example from Uzan's review [5]:

"Teller (1948) first emphasized that Dirac hypothesis may be in conflict with paleontological evidence. His argument is based on the estimation of the temperature at the center of the Sun $T_{\odot} \propto G M_{\odot} / R_{\odot}$ using the virial theorem. The luminosity of the Sun is then proportional to the radiation energy gradient times the mean free path of a photon times the surface of the Sun, that is $L_{\odot} \propto T_{\odot}^{7} R_{\odot}^{7} M_{\odot}^{-2}$, hence concluding that $L_{\odot} \propto T_{\odot}^{7} M_{\odot}^{5}$. Computing the radius of the Earth orbit in Newtonian mechanics, assuming the conservation of angular momentum (so that $G M_{\odot} R_{\text {Earth }}$ is constant) and stating that the Earth mean temperature is proportional to the fourth root of the energy received, he concluded that

$$
T_{\text {Earth }} \propto G^{2.25} M_{\odot}^{1.75}
$$

If $M_{\odot}$ is constant and $G$ was $\mathbf{1 0 \%}$ larger 300 million years ago, the Earth surface temperature should have been $20 \%$ higher, that is close to the boiling temperature. This was in contradiction with the existence of trilobites in the Cambrian".

Moreover, Teller didn't take the "Faint Young Sun” paradox into account: the young Sun’s output was only about $70 \%$ of what it is today [1]. So, all conclusions on the (almost) constancy of the Newtonian parameter of gravitation are model-dependent.

\section{Cosmological Parameters}

The advantage of WUM is that two fundamental parameters in various rational exponents define all macro and micro features of the World: Fine-structure constant $\alpha$, and dimensionless quantity $Q$. While $\alpha$ is constant, $Q$ increases with time, and is in fact a measure of the size and the age of the World, as well as all other time-varying parameters of the World [1]-[3]. $Q$ can be calculated based on the value of the gravitational parameter $G$ :

$$
Q=\frac{a^{2} c^{4}}{8 \pi h c} \times G^{-1}
$$

Then all time-varying cosmological parameters can be calculated based on the value of $G$ :

- Hubble’s parameter $H$

$$
H=\frac{C}{a} \times Q^{-1} \propto G
$$

- Age of the World $A_{\tau}$

$$
A_{\tau}=\frac{a}{c} \times Q \propto G^{-1}
$$

- $\quad$ Size of the World $R$

$$
R=a \times Q \propto G^{-1}
$$

- Critical energy density $\rho_{c r}$

$$
\rho_{\text {cr }}=3 \rho_{0} \times Q^{-1} \propto G
$$

- Temperature of the microwave background radiation $T_{M B R}$

$$
T_{M B R}=\frac{E_{0}}{k_{B}}\left(\frac{15 \alpha}{2 \pi^{3}} \frac{m_{e}}{m_{p}}\right)^{1 / 4} \times Q^{-1 / 4} \propto G^{1 / 4}
$$

- Temperature of the far-infrared background radiation peak $T_{F I R B}$

$$
T_{F I R B}=\frac{E_{0}}{k_{B}}\left(\frac{15}{4 \pi^{5}}\right)^{1 / 4} \times Q^{-1 / 4} \propto G^{1 / 4}
$$


- Planck mass $M_{P}$

$$
M_{P}=2 m_{0} \times Q^{1 / 2} \propto G^{-1 / 2}
$$

- Electronic neutrino mass $m_{v_{e}}$

$$
m_{v_{e}}=\frac{1}{24} m_{0} \times Q^{-1 / 4} \propto G^{1 / 4}
$$

- Muonic neutrino mass $m_{v_{\mu}}$

$$
m_{v_{\mu}}=m_{0} \times Q^{-1 / 4} \propto G^{1 / 4}
$$

- Tauonic neutrino mass $m_{v_{\tau}}$

$$
m_{v_{\tau}}=6 m_{0} \times Q^{-1 / 4} \propto G^{1 / 4}
$$

- Axion mass $m_{a}$

$$
m_{a}=\left(\frac{m_{e}}{m_{p}}\right)^{1 / 2} \times m_{0} \times Q^{-1 / 2} \propto G^{1 / 2}
$$

where $k_{\mathrm{B}}$ is the Boltzmann constant, $\rho_{0}$ is a basic unit of energy density:

$$
\rho_{0}=\frac{h c}{a^{4}}
$$

and $m_{0}$ is a basic unit of mass:

$$
m_{0}=\frac{h}{a c}=70.025267 \mathrm{MeV} / \mathrm{c}^{2}
$$

As shown in [1]-[3], the calculated values of these parameters are in a good agreement with the latest results of their measurements. For example, calculating the value of Hubble's parameter $H_{0}$ based on $G$ we find

$$
H_{0}=\frac{8 \pi h c}{a^{3} c^{3}} \times G=68.7457(83) \frac{\mathrm{km} / \mathrm{s}}{\mathrm{Mpc}}
$$

which is in good agreement with $H_{0}=69.32 \pm 0.8 \frac{\mathrm{km} / \mathrm{s}}{\mathrm{Mpc}}$ obtained using WMAP data [7].

We can calculate the value of $T_{M B R}$ (see 3.6) and get $T_{M B R}=2.72518 \mathrm{~K}$ which is in excellent agreement with experimentally measured value of $2.72548 \pm 0.00057 \mathrm{~K}$ [8].

In frames of WUM, some cosmological parameters are constants and can be calculated based on the value of the fine-structure constant $\alpha$. WUM postulates that masses of Dark Matter Particles (DMP) are proportional to $m_{0}$ multiplied by different exponents of $\alpha$ [2]:

Cold DMP (neutralinos and WIMPs):

$$
\begin{gathered}
m_{N}=\alpha^{-2} m_{0}=1.3149950 \mathrm{TeV} / \mathrm{c}^{2} \\
m_{\text {WIMP }}=\alpha^{-1} m_{0}=9.5959823 \mathrm{GeV} / \mathrm{c}^{2}
\end{gathered}
$$

DIRACs:

$$
m_{\text {DIRAC }}=2 \alpha^{0} \frac{m_{0}}{2}=70.025267 \mathrm{MeV} / \mathrm{c}^{2}
$$

ELOPs:

$$
m_{E L O P}=2 \alpha^{1} \frac{m_{0}}{3}=340.66606 \mathrm{keV} / \mathrm{c}^{2}
$$

Warm DMP (sterile neutrinos):

$$
m_{v_{s}}=\alpha^{2} m_{0}=3.7289402 \mathrm{keV} / \mathrm{c}^{2}
$$


These values fall into the mass ranges estimated in literature [2]. The roles of those particles in macroobject cores built up from fermionic dark matter, in gamma-ray spectra of the diffuse gamma-ray background and the emission of various macroobjects in the World are discussed in [2].

One of the principal ideas of WUM holds that relative energy densities of the World's particles in terms of the critical energy density $\rho_{c r}$ are constants in all times; depend only on the fundamental parameter $\alpha$ and proportional to proton energy density in the World's Medium [1]:

$$
\Omega_{p}=\frac{2 \pi^{2} \alpha}{3}=0.048014655
$$

The relative energy densities of the components of the World are:

Protons $\Omega_{\text {ptot }}$

$$
\Omega_{p t o t}=1.5 \Omega_{p}=\pi^{2} \alpha
$$

Electrons $\Omega_{\text {etot }}$

$$
\Omega_{\text {etot }}=1.5 \frac{m_{e}}{m_{p}} \Omega_{p}=\frac{m_{e}}{m_{p}} \pi^{2} \alpha
$$

Microwave background radiation $\Omega_{M B R}$

$$
\Omega_{M B R}=3 \frac{m_{e}}{m_{p}} \Omega_{p}=2 \frac{m_{e}}{m_{p}} \pi^{2} \alpha
$$

Dark Matter $\Omega_{D M t o t}$

$$
\Omega_{D M t o t}=5 \Omega_{p}=\frac{10}{3} \pi^{2} \alpha=0.24007328
$$

Cosmic Neutrino Background $\Omega_{C N B}$

$$
\Omega_{C N B}=\frac{45}{\pi} \Omega_{p}=30 \pi \alpha=0.68775927
$$

Dineutrinos $\Omega_{v \bar{V}}$

$$
\Omega_{v \bar{v}}=3 \frac{m_{e}}{m_{p}} \Omega_{p}=2 \frac{m_{e}}{m_{p}} \pi^{2} \alpha
$$

Far-infrared background radiation $\Omega_{\text {FIRB }}$

$$
\Omega_{F I R B}=\frac{3}{10 \pi} \frac{m_{e}}{m_{p}} \Omega_{p}=\frac{1}{5 \pi} \frac{m_{e}}{m_{p}} \pi^{2} \alpha
$$

The sum of all components densities of the World $\Omega_{W}$ is

$$
\begin{aligned}
\Omega_{W} & =\Omega_{p t o t}+\Omega_{e t o t}+\Omega_{M B R}+\Omega_{D M}+\Omega_{C N B}+\Omega_{\nu \bar{v}}+\Omega_{F I R B} \\
& =\left[\frac{45}{\pi}+6.5+\left(5.5+\frac{1}{5 \pi}\right) \frac{m_{e}}{m_{p}}\right] \frac{2 \pi^{2} \alpha}{3}=1
\end{aligned}
$$

in all times. The implication is that the World is flat.

From (3.29) we can calculate the value of $\alpha$, using electron-to-proton mass ratio $\frac{m_{e}}{m_{p}}$

$$
\frac{1}{\alpha}=\frac{\pi}{15}\left[450+65 \pi+(55 \pi+2) \frac{m_{e}}{m_{p}}\right]=137.03600
$$

which is in excellent agreement with the commonly adopted value of $137.035999074(44)$. It means that $\frac{m_{e}}{m_{p}}$ is not an independent constant, but is instead derived from $\alpha$ [3]. 
With the exception of neutrinos, the calculated values of the energy densities of the components of the World are in good agreement with their latest measurements [1]-[3]. When it comes to neutrinos, WUM postulates a much higher energy density than is commonly accepted in literature. As we proceed to show in the next section, there is no need for Dark Energy in WUM.

\section{Gravitational Effects}

The very first gravitational effect was calculated by J. G. von Soldner in 1801. In his paper "The deflection of a light ray from its rectilinear motion, by the attraction of a celestial body at which it nearly passes by" he found for the angle of deflection by Sun $\theta$ the value $\theta=0.84 \mathrm{arcsec}$ which is very close to the value $\theta=0.87$ arcsec calculated by Einstein in 1908 [9]. And only in 1915 Einstein presented the $\theta=1.75$ arcsec calculation based on General Theory of Relativity.

In our opinion, there is another possibility to explain an increased value of the deflection angle by Sun. According to WUM, all macroobjects of the World have cores made up of fermionic DMP. In case of extrasolar systems, the cores of stars are made up of interacting neutralinos or WIMPs surrounded with white dwarf shells.

Surrounding the cores, there is a transitional region in which the density decreases rapidly to the point of the zero level of the fractal structure [10] characterized by radius $R_{f}$ and energy density $\rho_{f}$ that satisfy the following equation for $r \geq R_{f}$ :

$$
\rho(r)=\frac{\rho_{f} R_{f}}{r}
$$

The transition region between solar core and the beginning of the Heliosphere, in which the density considerably decreases, may cause an additional deflection of a light ray due to the gravitational refraction.

A gravitational lens refers to a distribution of matter (such as a cluster of galaxies) between a distant source and an observer that is capable of bending the light from the source, as it travels towards the observer. Fritz Zwicky posited in 1937 that the effect could allow galaxy clusters to act as gravitational lenses. It was not until 1979 that this effect was confirmed by observation of the so-called "Twin QSO" SBS $0957+561$.

According to WUM, sterile neutrinos make up cores of galaxy clusters. The cores are surrounded by shells made up of DM and baryonic matter. Every macroobject consists of all particles under consideration that are present in the same proportion as they exist in the World's Medium [2].

In our opinion, the structure of galaxy clusters described above should be taken into account whenever gravitational lenses are calculated.

Gravitational redshift is the process by which electromagnetic radiation originating from a source that is in a gravitational field is reduced in frequency, or redshifted, when observed in a region of a weaker gravitational field. This effect is now considered to have been definitively verified by the experiments of Pound, Rebka and Snider between 1959 and 1965.

The gravitational redshift depends on the mass of the gravitating body. WUM holds that $1 / 3$ of the total mass is in the central macroobject (for example, a star in extrasolar system) and 2/3 of the total mass is in the fractal structure around it [1]. This mass ratio should be taken into account when calculating gravitational redshift.

The gravitational redshift is a part of the total cosmological redshift. Let us analyze the movement of photons as they travel from distant galaxies to Earth in the time-varying Medium. As we have shown in [1], energy of photons remains constant in the ideal frictionless Medium. In the actual rotationally elastic Medium [11] with a friction coefficient for photons

$$
k_{p h} \sim \tau^{-1}
$$

the equation for the photons momentum $p_{p h}$ is:

$$
\frac{\mathrm{d} p_{p h}}{\mathrm{~d} \tau}=-\delta \frac{p_{p h}}{\tau}
$$

where $\delta$ is a parameter. Solving equation 4.3 we obtain

$$
p_{p h} \tau^{\delta}=\text { const }
$$

Consider a photon with initial momentum $p_{\text {emit }}$ emitted at time $\tau_{\text {emit }}$. The photon is continuously losing 
momentum as it moves through the Medium until time $\tau_{\text {obsv }}$ when it is observed. The observer will measure $\lambda_{\text {obsv }}$, compare it with well-known wavelength $\lambda_{\text {emit }}$, and calculate a redshift:

$$
z=\frac{\lambda_{\text {obsv }}-\lambda_{\text {emit }}}{\lambda_{\text {emit }}}
$$

By definition, $\lambda=\frac{h}{p}$. When $\delta=1$ we obtain:

$$
\begin{gathered}
p_{\text {obsv }} \tau_{\text {obsv }}=p_{\text {emit }} \tau_{\text {emit }} \\
1+z=\frac{\lambda_{\text {obsv }}}{\lambda_{\text {emit }}}=\frac{p_{\text {emit }}}{p_{\text {obsv }}}=\frac{\tau_{\text {obsv }}}{\tau_{\text {emit }}}
\end{gathered}
$$

Recall that $\tau_{\text {emit }}$ and $\tau_{\text {obsv }}$ are cosmological times (ages of the World at the moments of emitting and observing), both measured from the Beginning of the World. $\tau_{\text {obsv }}$ equals to the present age of the World $A_{\tau}$. If the photon travelled for time $t_{p h}$, then

$$
\begin{gathered}
\tau_{\text {obsv }}=\tau_{\text {emit }}+t_{p h} \\
t_{p h}=\tau_{\text {obsv }}-\tau_{\text {emit }}=A_{\tau}-t_{\text {emit }}
\end{gathered}
$$

The cosmological redshift is then described by a nonlinear equation on $t_{p h}$ :

$$
1+z=\frac{1}{1-t_{p h} / A_{\tau}}
$$

As an example, a photon travelling for 7.11 Byr (half of the World's age $A_{\tau}$ ) will have a redshift of $1+z=2$. Photon travelling for $12.64 \mathrm{Byr}$ will have a redshift of $1+z=9$. The difference is due to the dependence of the Medium friction on time: it was 9 times greater at $\tau_{\text {emit }}=1.58 \mathrm{Byr}$ than it is now at $\tau_{\text {obsv }} \approx 14.22 \mathrm{Byr}$.

In accordance with Hubble's law, the distance $d$ to galaxies for $z \ll 1$ is found to be proportional to $z$ :

$$
d=\frac{c}{H_{0}} z=R z
$$

The relationship of distance $d$ to the redshift $z$ for large values of $z$ is not presently conclusive, active research is conducted in the area. In our Model, the distance to galaxies equals to:

$$
d=\frac{C}{H} \frac{z}{1+z}=R \frac{Z}{1+z}
$$

which reduces to 4.11 for $z \ll 1$ and $d=R$ for $z \rightarrow \infty$.

Experimental observations measuring light from distant galaxies and supernovae seem to imply that the World is expanding at an accelerated pace, as is evident from the observed redshift. Since 1990s, Dark Energy became the widely accepted hypothesis that explains this phenomenon.

The time varying friction of the Medium offered above provides an alternative interpretation of these observations. For $z>1$, the distance to supernovae is smaller than expected and hence supernovae are brighter. There is then no reason to introduce dark energy in order to explain the nonlinear relationship of distance to the redshift.

In WUM the theoretical need for additional energy density distinct from the baryon matter and dark matter densities to form our observationally flat World is satisfied with the considerably larger fraction of the neutrino energy density in the total energy density of the World (see 3.26). Consequently, we are dealing with wellknown particles instead of dark energy.

The idea of loss of energy of the photon in the intergalactic medium was first suggested in 1929 by Zwicky. But there are two problems: 1) all images of distant objects look blurred if the intergalactic space produces scattering; 2) the scattering effect and the consequent loss of energy is frequency dependent [12].

Different mechanisms were proposed to avoid blurring and scattering. Laio A., et al. showed that the shift of photon frequency in low density plasma (which is the case in our Model [1]) could come from quantum effects derived from standard quantum electrodynamics [13]. According to E. J. Lerner, quantum mechanics indicates that a photon gives up a tiny amount of energy as it collides with an electron, but its trajectory does not change [14]. 
There is another way to explain the absence of the blurring and scattering. Back in 1846 James McCullagh proposed a theory of rotationally elastic medium, i.e. the medium in which every particle resists absolute rotation [11]. This theory produces equations analogous to Maxwell's electromagnetic equations. In our opinion, the Medium of the World is in fact such a rotationally elastic medium. We propose to review the interaction of photons with the Medium in light of this unique theory.

\section{Gravitoelectromagnetism}

Gravitoelectromagnetism (GEM) refers to a set of formal analogies between the equations for electromagnetism and relativistic gravitation. GEM is an approximation to the Einstein field equations for general relativity in the weak field limit. The equations for GEM were first published in 1893, before general relativity, by O. Heaviside as a separate theory expanding Newton's law [15]. WUM follows this theory.

Maxwell's equations (ME) vary with the unit system used. Although the general shape remains the same, various definitions are changed, and different constants appear in different places. We'll start our discussion with ME in SI units. We will not rewrite well-known equations, but only provide the relationships between physical quantities used in ME for electromagnetism and gravitoelectromagnetism in the Table 2 and Table 3:

In Maxwell's equations, electrodynamic constant $c$ is defined as the ratio of the absolute electromagnetic unit of charge to the absolute electrostatic unit of charge.

From Table 2 and Table 3 it becomes clear that the dimensions of all physical quantities depend on the choice of the charge and mass dimensions (Coulomb \& kilogram in SI units). In other unit systems the dimensions are different. For instance, in Gaussian units (CGSE):

\section{Table 2. Electromagnetism.}

\begin{tabular}{ccc} 
Charge & Impedance of Electromagnetic Field & Magnetic Flux \\
$q, \mathrm{C}$ & $Z_{0}=\sqrt{\frac{\mu_{0}}{\varepsilon_{0}}}=\mu_{0} c, \Omega$ & $\phi_{q}, \mathrm{~Wb}$ \\
Electric Current & Magnetic Constant & Electric Potential \\
$I_{q}, \mathrm{~A}$ & $\mu_{0}, \mathrm{H} \cdot \mathrm{m}^{-1}$ & $U_{q}, \mathrm{~V}$ \\
Magnetic Field Intensity & Electric Constant & Electric Field \\
$\boldsymbol{H}_{q}, \mathrm{~A} \cdot \mathrm{m}^{-1}$ & $\varepsilon_{0}=\left(\mu_{0} \mathrm{c}^{2}\right)^{-1}, \phi \cdot \mathrm{m}^{-1}$ & $\boldsymbol{E}_{q}, \mathrm{~V} \cdot \mathrm{m}^{-1}$ \\
Electric Flux Density & Electrodynamic Constant & Magnetic Flux Density \\
$\boldsymbol{D}_{q}, \mathrm{C} \cdot \mathrm{m}^{-2}$ & $c, \mathrm{~m} \cdot \mathrm{s}^{-1}$ & $\boldsymbol{B}_{q}, \mathrm{~Wb} \cdot \mathrm{m}^{-2}$ \\
\hline
\end{tabular}

Table 3. Gravitoelectromagnetism.

\begin{tabular}{ccc} 
Mass & Impedance of Gravitational Field & Gravitomagnetic Flux \\
$m, \mathrm{~kg}$ & $Z_{g}=\sqrt{\frac{\mu_{g}}{\varepsilon_{g}}}=\mu_{g} c$ & $\phi_{m}, \mathrm{~m}^{2} \cdot \mathrm{s}^{-1}$ \\
Mass Current & Gravitomagnetic Parameter & Gravitoelectric potential \\
$I_{m}, \mathrm{~kg} \cdot \mathrm{s}^{-1}$ & $\mu_{g}=\frac{4 \pi G}{c^{2}}$ & $U_{m}, \mathrm{~m}^{2} \cdot \mathrm{s}^{-2}$ \\
Gravitomagnetic Field Intensity & Gravitoelectric Parameter & Gravitoelectric Field \\
$\boldsymbol{H}_{m}, \mathrm{~kg} \cdot \mathrm{m}^{-1} \cdot \mathrm{s}^{-1}$ & $\varepsilon_{g}=\left(\mu_{g} c^{2}\right)^{-1}$ & $\boldsymbol{E}_{m}, \mathrm{~m} \cdot \mathrm{s}^{-2}$ \\
Gravitoelectric Flux Density & Gravitoelectrodynamic Constant & Gravitomagnetic Flux Density \\
$\boldsymbol{D}_{m}, \mathrm{~kg} \cdot \mathrm{m}^{-2}$ & $c, \mathrm{~m} \cdot \mathrm{s}^{-1}$ & $\boldsymbol{B}_{m}, \mathrm{~s}^{-1}$ \\
\hline
\end{tabular}


- $\left[q_{e}\right]=\mathrm{cm}^{3 / 2} \cdot \mathrm{g}^{1 / 2} \cdot \mathrm{s}^{-1}$

- $\left[Z_{e}\right]=\mathrm{cm}^{-1} \cdot \mathrm{s}$ In CGSM:

- $\left[q_{m}\right]=\mathrm{cm}^{1 / 2} \cdot \mathrm{g}^{1 / 2}$

- $\left[Z_{m}\right]=\mathrm{cm} \cdot \mathrm{s}^{-1}$

We seem to possess a substantial degree of freedom when it comes to choosing the dimension of charge. For an arbitrary dimension-transposing parameter $P$ we can

- Multiply the charge and mass and all physical quantities on the left side of Table 2 and Table 3 by an arbitrary parameter $P$,

- Divide impedances by $P^{2}$,

- Divide magnetic fluxes and all physical quantities on the right side of Table 2 and Table 3 by $P$.

Following such a transformation, all physically measurable parameters such as energy density and energy flux density remain the same, and have the same mechanical dimensions.

By definition, 1 Coulomb equals to one tenth of the absolute electromagnetic unit of charge. It follows that in SI we use electromagnetic unit of charge $e$ in the electrostatic Coulomb law instead of the electrostatic unit $\frac{e}{c}$. This seems a bit odd.

Likewise, when describing Newtonian law of gravitation, we use $m$ - the inertial mass, instead of gravitoelectrostatic charge $m c$-the gravitational mass. The gravitoelectromagnetic charge is then $m c^{2}$. Similarly to the electromagnetic field, the gravitoelectrodynamic constant $c$ is the ratio of the absolute gravitoelectromagnetic unit of charge to the absolute gravitoelectrostatic unit of charge.

All elementary particles in the World are fully characterized by their four-momentum $\left(\frac{E}{c}, \boldsymbol{p}\right)$ that satisfies the following equation:

$$
\left(\frac{E}{c}\right)^{2}-\boldsymbol{p}^{2}=\operatorname{Inv}=(m c)^{2}
$$

where the invariant is, in fact, the gravitoelectrostatic charge $m c$ squared, and $E$ is the gravitoelectromagnetic charge.

The inertial mass and the gravitational mass are not the same physical quantity. Instead, they are proportional to each other, and their ratio equals to the gravitoelectrodynamic constant $c$. The classical theory offers no compelling reason why the gravitational mass $m c$ has to equal the inertial mass $m$, commonly referred to as "rest mass".

Analogous to electromagnetism, we can think of $m$ as a gravitocapacitor. Then, $E=m c^{2}$ describes the accumulation of energy by gravitocapacitor with capacity $m$, rather than transformation of energy to mass.

But there is a principal physical difference between Electromagnetism (EM) and Gravitoelectromagnetism (GEM):

- In EM, the magnetic constant $\mu_{0}$ and electric constant $\varepsilon_{0}$ are the vacuum permeability and vacuum permittivity of free (empty) space correspondingly;

- In GEM, the gravitomagnetic parameter $\mu_{g}$ depends on the gravitational parameter $G$ :

$$
\mu_{g}=\frac{4 \pi G}{c^{2}}
$$

which is not a constant in our model and cannot be introduced without the Medium of the World. In frames of WUM the gravitomagnetic parameter $\mu_{g}$ can be calculated based on the value of the energy density of the Medium of the World $\rho_{M}$ :

$$
\mu_{g}=\frac{4 \pi G}{c^{2}}=\frac{\rho_{M}}{c^{2}} \times P^{2}
$$

where a dimension-transposing parameter $P$ equals to [1]:

$$
P=\frac{a^{3}}{2 h / c}
$$


Nikola Tesla stated the existence of the Medium of the World: "All attempts to explain the workings of the universe without recognizing the existence of the ether and the indispensable function it plays in the phenomena are futile and destined to oblivion".

James McCullagh has this to say about the Medium: "The constitution of the ether, if it ever would be discovered, will be found to be quite different from anything that we are in the habit of conceiving, though at the same time very simple and very beautiful. An elastic medium composed of points acting on each other in the way supposed by Poisson and others will not answer".

Long time ago it was realized that there are no longitudinal waves in the Medium, and hence the Medium could not be an elastic matter of an ordinary type. In 1846 James McCullagh proposed a theory of a rotationally elastic medium, i.e. a medium in which every particle resists absolute rotation [11].

The potential energy of deformation in such a medium depends only on the rotation of the volume elements and not on their compression or general distortion. This theory produces equations analogous to Maxwell's electromagnetic equations.

The World-Universe Model is based on Maxwell's equations, and McCullagh's theory is a good fit for description of the Medium.

As the conclusion:

- The gravitation does not exist without the Medium of the World;

- The gravitation is connected to the main characteristic of the Medium-energy density.

\section{Le Sage's Theory of Gravitation}

Wikipedia summarizes this unique theory as follows:

"Le Sage's theory of gravitation is a kinetic theory of gravity originally proposed by Nicolas Fatio de Duillier in 1690 and later by Georges-Louis Le Sage in 1748. The theory proposed a mechanical explanation for Newton's gravitational force in terms of streams of tiny unseen particles (which Le Sage called ultra-mundane corpuscles) impacting all material objects from all directions. According to this model, any two material bodies partially shield each other from the impinging corpuscles, resulting in a net imbalance in the pressure exerted by the impact of corpuscles on the bodies, tending to drive the bodies together".

Le Sage proposed quantitative estimates for some of the theory's parameters:

- He called the gravitational particles ultramundane corpuscles, because he supposed them to originate beyond our known universe. The distribution of the ultramundane flux is isotropic and the laws of its propagation are very similar to that of light.

- He suggested that the ultramundane corpuscles might move at the speed of light.

- To maintain mass proportionality, ordinary matter consists of cage-like structures, in which their diameter is only the $10^{7}$ th part of their mutual distance, so the particles can travel through them nearly unhindered.

Lyman Spitzer in 1941 calculated that absorption of radiation between two dust particles lead to a net attractive force which varies proportional to $1 / \mathrm{r}^{2}[16]$.

The Le Sage mechanism also has been identified as a significant factor in the behavior of dusty plasma. A. M. Ignatov has shown that an attractive force arises between two dust grains suspended in isotropic collisionless plasma due to inelastic collisions between ions of the plasma and the grains of dust. This attractive force is inversely proportional to the square of the distance between dust grains, and can counterbalance the Coulomb repulsion between dust grains [17].

Although it is not regarded as a viable theory within the mainstream scientific community, there are some attempts to re-habilitate the theory [18]-[25]. In this respect, we would like to stress the importance of the extended theories of gravity in the debate about gravitation, as it is clarified in [26].

Every Le Sage-type model assumes the existence of a space-filling isotropic flux or radiation of enormous intensity and penetrating capability. The flux of neutrinos emanating from the Sun was discussed in literature. This flux possesses the penetrating properties envisaged by Le Sage, but it is not isotropic, and its intensity is even smaller than that of the Cosmic Microwave Background radiation.

In our model, the Cosmic Neutrino Background (CNB) is indeed a space-filling and fairly isotropic flux. It has a high intensity since its total neutrino energy density $\Omega_{C N B}$ is about $69 \%$ of the total energy density of the World $\Omega_{W}$ (see 3.26). One may wonder-if there are so many neutrinos out there, how come the numerous neutrino detectors do not register them in significant quantities? 
According to WUM, CNB consists of three different types of neutrinos: electronic $v_{e}$, muonic $v_{\mu}$, and tauonic $v_{\tau}$, and their antiparticles with masses $m_{v_{e}}, m_{v_{\mu}}, m_{v_{\tau}}$ [3]:

$$
\begin{gathered}
m_{v_{e}}=\frac{1}{24} m_{0} \times Q^{-1 / 4} \cong 3.1 \times 10^{-4} \mathrm{eV} / \mathrm{c}^{2} \\
m_{v_{\mu}}=m_{0} \times Q^{-1 / 4} \cong 7.5 \times 10^{-3} \mathrm{eV} / \mathrm{c}^{2} \\
m_{v_{\tau}}=6 m_{0} \times Q^{-1 / 4} \cong 4.5 \times 10^{-2} \mathrm{eV} / \mathrm{c}^{2}
\end{gathered}
$$

For Fermi momentum $p_{F}$ we took the following value [3]:

$$
p_{F}^{2}=\frac{m_{0}^{2} c^{2}}{2 \pi^{2}} \times Q^{-1 / 2}
$$

Then for Fermi energy $E_{F}$ we obtain:

$$
\begin{gathered}
E_{F v_{e}}=p_{F} c=\frac{1}{\sqrt{2} \pi} m_{0} c^{2} \times Q^{-1 / 4} \cong 7.2 k_{B} T_{M B R} \\
E_{F v_{\mu}}=\frac{p_{F}^{2}}{2 m_{v_{\mu}}}=\frac{1}{4 \pi^{2}} m_{0} c^{2} \times Q^{-1 / 4} \cong 0.81 k_{B} T_{M B R} \\
E_{F v_{\tau}}=\frac{p_{F}^{2}}{2 m_{v_{\tau}}}=\frac{1}{24 \pi^{2}} m_{0} c^{2} \times Q^{-1 / 4} \cong 0.135 k_{B} T_{M B R}
\end{gathered}
$$

It follows that CNB consists of very low-energy neutrinos, whose energy is similar to that of the Cosmic Microwave Background radiation. Their interaction with matter is very weak. Since the neutrino-induced crosssections depend on the neutrinos energy linearly, such background neutrinos will not be registered by standard neutrino detectors. In fact, we might never be able to directly observe the CNB.

The obtained results show that the proposed CNB mechanism of Gravitation is relevant for the Le Sage's theory.

In our model, Dark Matter particles (DMP) are a space-filling and fairly isotropic flux as well. It possesses the penetrating properties envisaged by Le Sage for his ultramundane corpuscles, and has a high intensity since the total DMP energy density $\Omega_{D M}$ is about $24 \%$ of the $\Omega_{W}$ (see 3.25).

We should recall that $1 / 3$ of the World energy $E_{W}$ is in all Macroobjects and 2/3 of $E_{W}$ is in the Medium of the World which is a space-filling and fairly isotropic in our model [1] and is responsible for the Le Sage's mechanism of the gravitation.

According to WUM, all material objects of the World have gravitational charges. Two particles or microobjects will not exert gravity on one another when both of their masses are smaller than the Planck mass. Planck mass can then be viewed as the mass of the smallest macroobject capable of generating the gravitoelectromagnetic field, and serves as a natural borderline between classical and quantum physics [3].

It is obvious that for the realization of Le Sage's mechanism of gravitation at least one material object must be a macroobject. In our opinion, the smallest such macroobject has Planck mass. The validity of this statement follows from the work of Lyman Spitzer [16] and A. M. Ignatov [17] who identified Le Sage's mechanism as a significant factor in the behavior of dust particles and dusty plasma.

As the conclusion:

- Gravity is not an interaction but a manifestation of the Medium of the World;

- Le Sage's theory is the very first theory which defines the Gravity as an emergent phenomenon.

\section{Emergent Gravity, Space and Time}

By definition, an emergent phenomenon is a property that is a result of simple interactions that work cooperatively to create a more complex interaction. Physically, the simple interactions occur at a microscopic level, and the collective result can be observed at a macroscopic level. In Le Sage's theory the gravity is just a result of mi- 
croscopic interactions which appear to average out on a macroscopic scale and give us gravity as we recognize it.

C. Barcelo, S. Liberati, and M. Visser have this to say about emergent gravity:

"One of the more fascinating approaches to 'quantum gravity' is the suggestion, typically attributed to Sakharov [27] [28] that gravity itself may not be 'fundamental physics'. Indeed it is now a relatively common opinion, maybe not mainstream but definitely a strong minority opinion, that gravity (and in particular the whole notion of spacetime and spacetime geometry) might be no more 'fundamental' than is fluid dynamics. The word 'fundamental' is here used in a rather technical sense-fluid mechanics is not fundamental because there is a known underlying microphysics that of molecular dynamics, of which fluid mechanics is only the low-energy low-momentum limit" [29].

With Albert Einstein's principle at heart- "When forced to summarize the theory of relativity in one sentence: time and space and gravitation have no separate existence from matter"-we introduced the Medium of the World consisting of protons, electrons, photons, neutrinos, and dark matter particles. In our model the Medium is not fundamental and has the macroscopic parameters like in fluid mechanics: impedance, gravitomagnetic parameter, etc.

In frames of WUM we can find the gravitomagnetic parameter of the Medium $\mu_{g}$ :

$$
\mu_{g}=\frac{4 \pi G}{c^{2}}=\frac{1}{R} \times P
$$

and the impedance of the Medium $Z_{g}$ :

$$
Z_{g}=\mu_{g} c=H \times P=\frac{1}{\tau} \times P
$$

where $R$ is the size of the World, $H$ is Hubble's parameter and $\tau$ is the absolute cosmological time measured from the Beginning of the World like absolute temperature measured from absolute zero in kelvins.

It follows that measuring the value of Hubble's parameter anywhere in the World and taking its inverse value allows us to calculate the absolute time of the World $\tau$. The Hubble's parameter is then the most important characteristic of the World, as it defines the Worlds' age.

The second important characteristic of the World is the gravitomagnetic parameter. Taking its inverse value, we can find the absolute size of the World $R$. We emphasize that the above two parameters $\left(Z_{g}\right.$ and $\mu_{g}$ ) are principally different physical characteristics of the Medium that are connected through the gravitoelectrodynamic constant $c$.

In WUM, time and space are closely connected with the Mediums' impedance and gravitomagnetic parameter. It follows that neither time nor space could be discussed in absence of the Medium. The gravitational parameter $G$ can be introduced only for the World filled with matter. Matter, then, is primary to time and space and gravity, as Einstein has postulated.

It follows that the gravity, space and time itself can be introduced only for the World filled with matter consisting of elementary particles which take part in simple interactions at a microscopic level. The collective result of their interactions can be observed at a macroscopic level. It means that Gravity, Space and Time are the emergent phenomena.

When in history of the World can we introduce the Medium of the World-a macroscopic notion? According to WUM, at the beginning when the size of the World was equal to $a$ and the extrapolated density $\rho_{c r 0}$ equaled to (see 3.5 at $Q=1$ )

$$
\rho_{\text {cr } 0}=3 \rho_{0}
$$

the extrapolated total amount of the surface energy of the World $E_{W 0}$ was equal to [1]

$$
E_{W 0}=\frac{6}{\pi} E_{0}
$$

which was sufficient to produce DIRACs and lighter particles only. The conditions for generating the very first ensemble of particles and the first objects actualized when the size of the World $a_{M}$ was about the Bohr radius multiplied by $2 \pi$ (see 3.4 )

$$
a_{M}=\frac{a}{\alpha^{2}} \cong 3.3 \times 10^{-10} \mathrm{~m}
$$


at the cosmological time $\tau_{M}$ (see 3.3)

$$
\tau_{M}=\frac{1}{\alpha^{2}} \frac{a}{c} \cong 1.1 \times 10^{-18} \mathrm{~s}
$$

The total energy $E_{W M}$ was equal to

$$
E_{W M}\left(Q=\frac{1}{\alpha^{2}}\right)=\frac{6 E_{0}}{\pi \alpha^{4}}
$$

and the Planck mass was equal to twice the mass of WIMPs (see 3.8)

$$
M_{P}=2 m_{\text {WIMP }}=2 \frac{m_{0}}{\alpha}
$$

At that time, neutralinos (the heaviest particles in our model with mass $m_{N}=\frac{m_{0}}{\alpha^{2}}$ ) could initiate a gravitational collapse of all particles heavier than $2 m_{0}$ (neutralinos, WIMPs, protons) [3] with the resulting microobjects-nuclei. All lighter particles would then be attracted to the nuclei, increasing their masses and initiating the macroobjects' formation.

As the conclusion:

- The macroscopic notion - the Medium of the World can be introduced at the cosmological time $\tau_{M}$.

- The emergent Gravity, Space and Time can be introduced for cosmological times $\tau \geq \tau_{M}$.

While the Model needs significant further elaboration, it can already serve as a basis for a new physics proposed by Le Sage, J. McCullagh, O. Heaviside, P. Dirac, A. D. Sakharov, and P. Wesson.

\section{Acknowledgements}

I am grateful to the anonymous referee for important remarks. Special thanks to my son Ilya Netchitailo who questioned every aspect of the paper and helped shape it to its present form.

\section{References}

[1] Netchitailo, V.S. (2015) 5D World-Universe Model. Space-Time-Energy. Journal of High Energy Physics, Gravitation and Cosmology, 1, 25-34. http://dx.doi.org/10.4236/jhepgc.2015.11003

[2] Netchitailo, V.S. (2015) 5D World-Universe Model. Multicomponent Dark Matter. Journal of High Energy Physics, Gravitation and Cosmology, 1, 55-71. http://dx.doi.org/10.4236/jhepgc.2015.12006

[3] Netchitailo, V.S. (2016) 5D World-Universe Model. Neutrinos. The World. Journal of High Energy Physics, Gravitation and Cosmology, 2, 1-18. http://dx.doi.org/10.4236/jhepgc.2016.21001

[4] Mohr, P.J., Taylor, B.N. and Newell, D.B. (2012) CODATA Recommended Values of the Fundamental Physical Constants: 2010. https://arxiv.org/abs/1203.5425

[5] Uzan, J.-P. (2002) The Fundamental Constants and Their Variation: Observational Status and Theoretical Motivations. http://arxiv.org/abs/hep-ph/0205340

[6] Uzan, J.-P. (2011) Varying Constants, Gravitation and Cosmology. Living Reviews in Relativity, 14, 2.

[7] Bennett, C.L., et al. (2013) Nine-Year Wilkinson Microwave Anisotropy Probe (WMAP) Observations: Final Maps and Results. http://arxiv.org/abs/1212.5225

[8] Fixsen, D.J. (2009) The Temperature of the Cosmic Microwave Background. http://arxiv.org/abs/0911.1955

[9] von Soldner, J.G. (1801) On the Deflection of a Light Ray from Its Rectilinear Motion, by the Attraction of a Celestial Body at Which It Nearly Passes by. Berliner Astronomisches Jahrbuch, 161-172.

[10] Baryshev, Yu.V. (2008) Field Fractal Cosmological Model as an Example of Practical Cosmology Approach. http://arxiv.org/abs/0810.0162

[11] McCullagh, J. (1846) An Essay towards a Dynamical Theory of Crystalline Reflexion and Refraction. Transactions of the Royal Irish Academy, 21, 17-50.

[12] Lopez-Corredoira, M. (2003) Observational Cosmology: Caveats and Open Questions in the Standard Model. https://arxiv.org/abs/astro-ph/0310214

[13] Laio, A., Rizzi, G. and Tartaglia, A. (1997) Quantum Theory of Frequency Shifts of an Electromagnetic Wave Interacting with a Plasma. Physical Review E, 55, 7457. http://dx.doi.org/10.1103/PhysRevE.55.7457 
[14] Lerner, E.J. (1991) The Big Bang Never Happened: A Startling Refutation of the Dominant Theory of the Origin of the Universe. Random House, Toronto.

[15] Heaviside, O. (1893) A Gravitational and Electromagnetic Analogy. The Electrician, 31, 281-282.

[16] Spitzer, L. (1941) The Dynamics of the Interstellar Medium. II. Radiation Pressure. The Astrophysical Journal, 94, 232-244. http://dx.doi.org/10.1086/144328

[17] Ignatov, A.M. (1996) Lesage Gravity in Dusty Plasma. Plasma Physics Reports, 22, 585-589.

[18] Radzievskii, V.V. and Kagalnikova, I.I. (1960) The Nature of Gravitation. Vsesoyuz. Astronom.-Geodezich. Obsch. Byull, 26, 3-14.

[19] Shneiderov, A.J. (1961) On the Internal Temperature of the Earth. Bollettino di Geofisica Teoricaed Applicata, 3 , 137-159.

[20] Buonomano, V. and Engel, E. (1976) Some Speculations on a Causal Unification of Relativity, Gravitation, and Quantum Mechanics. International Journal of Theoretical Physics, 15, 231-246. http://dx.doi.org/10.1007/BF01807095

[21] Adamut, I.A. (1982) The Screen Effect of the Earth in the TETG. Theory of a Screening Experiment of a Sample Body at the Equator Using the Earth as a Screen. Nuovo Cimento C, 5, 189-208.

[22] Jaakkola, T. (1996) Action at a Distance and Local Action in Gravitation: Discussion and Possible Solution of the Dilemma. Apeiron, 3, 61-75.

[23] Van Flandern, T. (1999) Dark Matter, Missing Planets and New Comets. 2nd Edition, North Atlantic Books, Berkeley, Chapters 2-4.

[24] Edwards, M.R. (2002) Pushing Gravity: New Perspectives on Le Sage’s Theory of Gravitation. C. Roy Keys Inc., Montreal.

[25] Edwards, M.R. (2007) Photon-Graviton Recycling as Cause of Gravitation. Apeiron, 14, 214-223.

[26] Corda, C. (2009) Interferometric Detection of Gravitational Waves: The Definitive Test for General Relativity. International Journal of Modern Physics D, D18, 2275-2282. http://dx.doi.org/10.1142/s0218271809015904

[27] Sakharov, A.D. (1968) Vacuum Quantum Fluctuations in Curved Space and the Theory of Gravitation. Soviet Physics, Doklady, 12, 1040-1041.

[28] Visser, M. (2002) Sakharov’s Induced Gravity: A Modern Perspective. Modern Physics Letters A, 17, 977-991. http://arxiv.org/abs/gr-qc/0204062 http://dx.doi.org/10.1142/s0217732302006886

[29] Barcelo, C., Liberati, S. and Visser, M. (2011) Analogue Gravity. Living Reviews in Relativity, $14,3$. http://dx.doi.org/10.12942/lrr-2011-3

\section{Submit or recommend next manuscript to SCIRP and we will provide best service for you:}

Accepting pre-submission inquiries through Email, Facebook, Linkedin, Twitter, etc

A wide selection of journals (inclusive of 9 subjects, more than 200 journals)

Providing a 24-hour high-quality service

User-friendly online submission system

Fair and swift peer-review system

Efficient typesetting and proofreading procedure

Display of the result of downloads and visits, as well as the number of cited articles

Maximum dissemination of your research work

Submit your manuscript at: http://papersubmission.scirp.org/ 